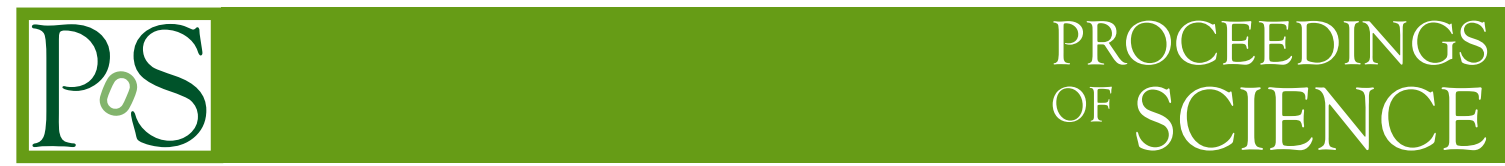

\title{
Kinematical correlations: from RHIC to LHC
}

\author{
Antoni Szczurek* \\ Institute of Nuclear Physics, PL-31-342 Cracow, Poland \\ and Univeristy of Rzeszów, PL-35-959 Cracow, Poland \\ E-mail: Antoni.Szczurek@ifj.edu.pl
}

Kinematical correlations between outgoing partons (elementary particles) are discussed in the framework of $k_{t}$-factorization. Different unintegrated gluon distributions are used. I discuss correlations between charm-anticharm, jets, photon and jet, and between leptons from semileptonic decays of heavy quarks as well as leptons produced in the Drell-Yan mechanism. Both correlations in azimuth as well as in the length of transverse momenta are shown. General conclusions are drawn.

High-pT Physics at LHC - Tokaj'08

16-19 March 2008

Tokaj, Hungary

${ }^{*}$ Speaker. 


\section{Introduction}

Most of the QCD calculations in the literature concentrated on calculating inclusive distributions (heavy quarks, jets, direct photons, etc). The standard collinear approach with next-to-leading order (NLO) accuracy is state of art in this respect. Higher luminosity and in the consequence better statistics at present and future colliders give a new possibility to study not only inclusive distributions but also correlations between outgoing partons or other elementary particles (photons, leptons, etc.). The standard collinear calculations are not the best suited for this purpose. In particular, one encounters singularities for back-to-back kinematics, i.e. when two elementary particles are emitted at angles differing in azimuth by $180^{\circ}$ or having the same length of the transverse momenta. At such configurations the standard collinear approach is not reliable and other methods should be used. The singularities can be avoided when initial transverse momenta of partons are included. In this presentation I discuss some general aspects within $k_{t}$-factorization approach, where so-called unintegrated gluon or parton distributions are used instead of usual standard distributions. The UGDFs (or UPDFs) are not only functions of longitudinal momentum fraction but also of (initial) transverse momenta. In general, there are two sources of initial transverse momenta. One is internal (Fermi) motion of hadron constituents. This, highly nonperturbative, effect is very difficult to calculate from first principles and therefore often neglected. The second is a perturbative effect related to parton evolution in QCD including $k_{t}$-kicks. The standard collinear approach limits itself somewhat arbitrarily to longitudinal directions of initial partons and neglects transverse degree of freedom.

Our group has calculated correlation observables for several processes:

- $c \bar{c}$ correlations [1],

- dijet correlations [2],

- photon-jet correlations [3]

- correlations of Drell-Yan dileptons and correlations of dilepton-jet [4].

In this talk I review the general strategy. The details can be found in original papers $[1,2,3,4]$. Only some selected detailed results are shown here. Some other results for $c \bar{c}$ and $e^{+} e^{-}$correlations were presented in parallel talks of my collaborators (see $[5,6])$ at this workshop.

\section{Sketch of the formalism}

In the $k_{t}$-factorization approach the cross section for the production of a pair of elementary particles $(k$ and $l$ ) can be written as

$$
\begin{aligned}
& \frac{d \sigma\left(h_{1} h_{2} \rightarrow k l\right)}{d^{2} p_{1, t} d^{2} p_{2, t}}=\sum_{i, j} \int d y_{1} d y_{2} \frac{d^{2} k_{1, t}}{\pi} \frac{d^{2} k_{2, t}}{\pi} \frac{1}{16 \pi^{2}\left(x_{1} x_{2} s\right)^{2}} \\
& \overline{|\mathscr{M}(i j \rightarrow k l)|^{2}} \delta^{2}\left(\vec{k}_{1, t}+\vec{k}_{2, t}-\vec{p}_{1, t}-\vec{p}_{2, t}\right) \mathscr{F}_{i}\left(x_{1}, k_{1, t}^{2}\right) \mathscr{F}_{j}\left(x_{2}, k_{2, t}^{2}\right),
\end{aligned}
$$

where

$$
x_{1}=\frac{m_{1, t}}{\sqrt{s}} \mathrm{e}^{+y_{1}}+\frac{m_{2, t}}{\sqrt{s}} \mathrm{e}^{+y_{2}},
$$




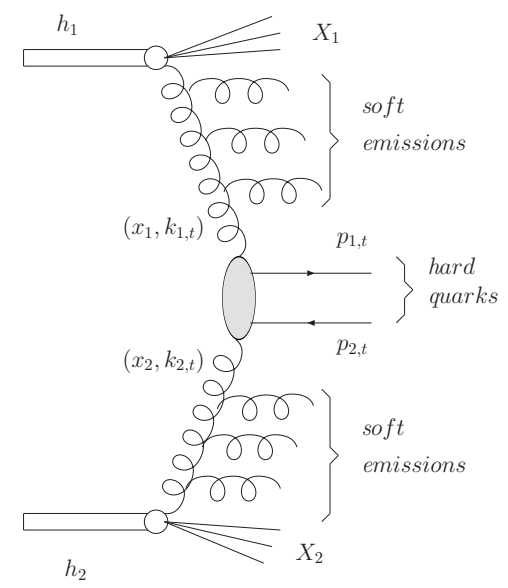

Figure 1: Diagram for $k_{t}$-factorization approach to $c \bar{c}$ production.
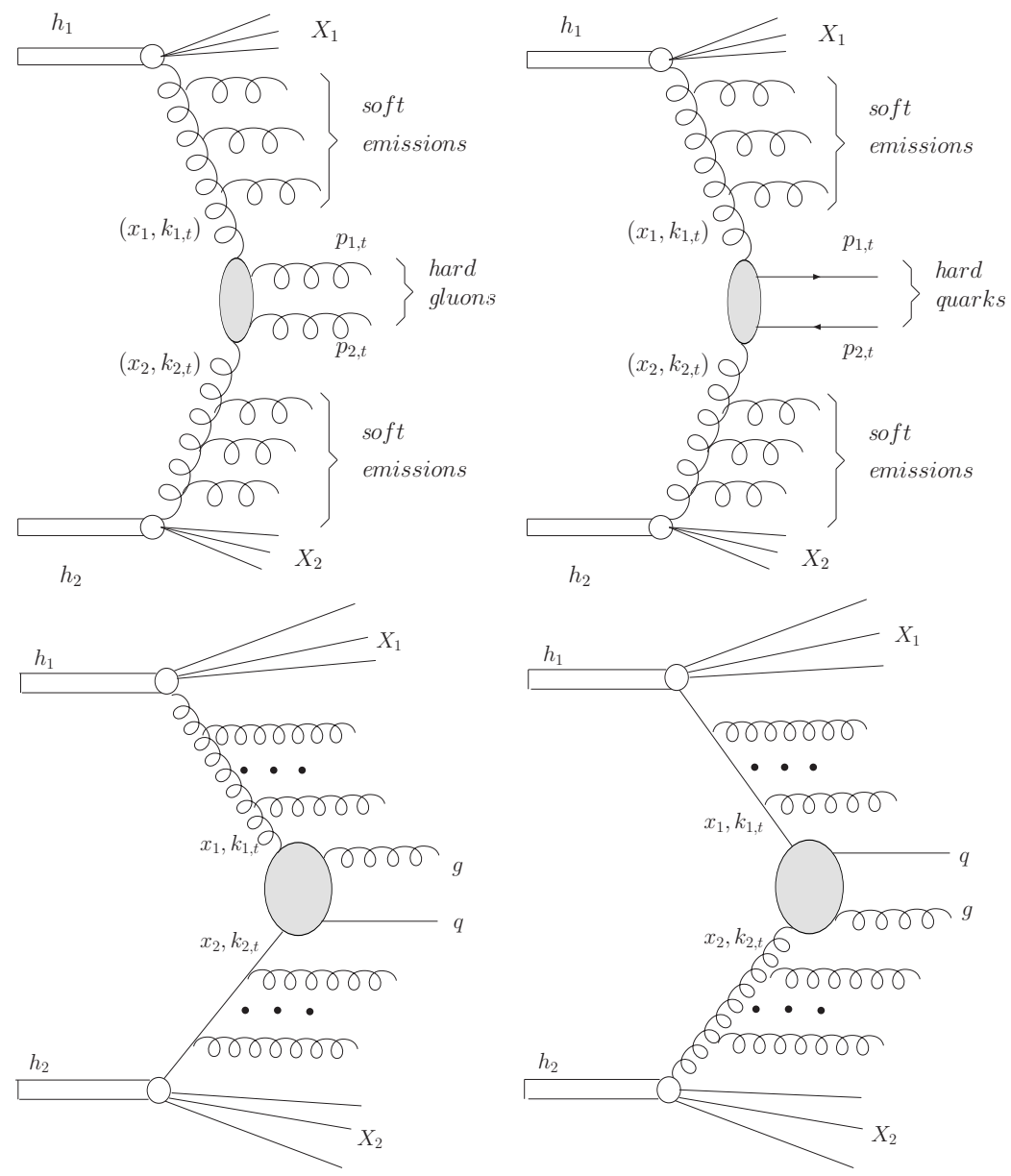

Figure 2: Diagrams for $k_{t}$-factorization approach to dijet production. 

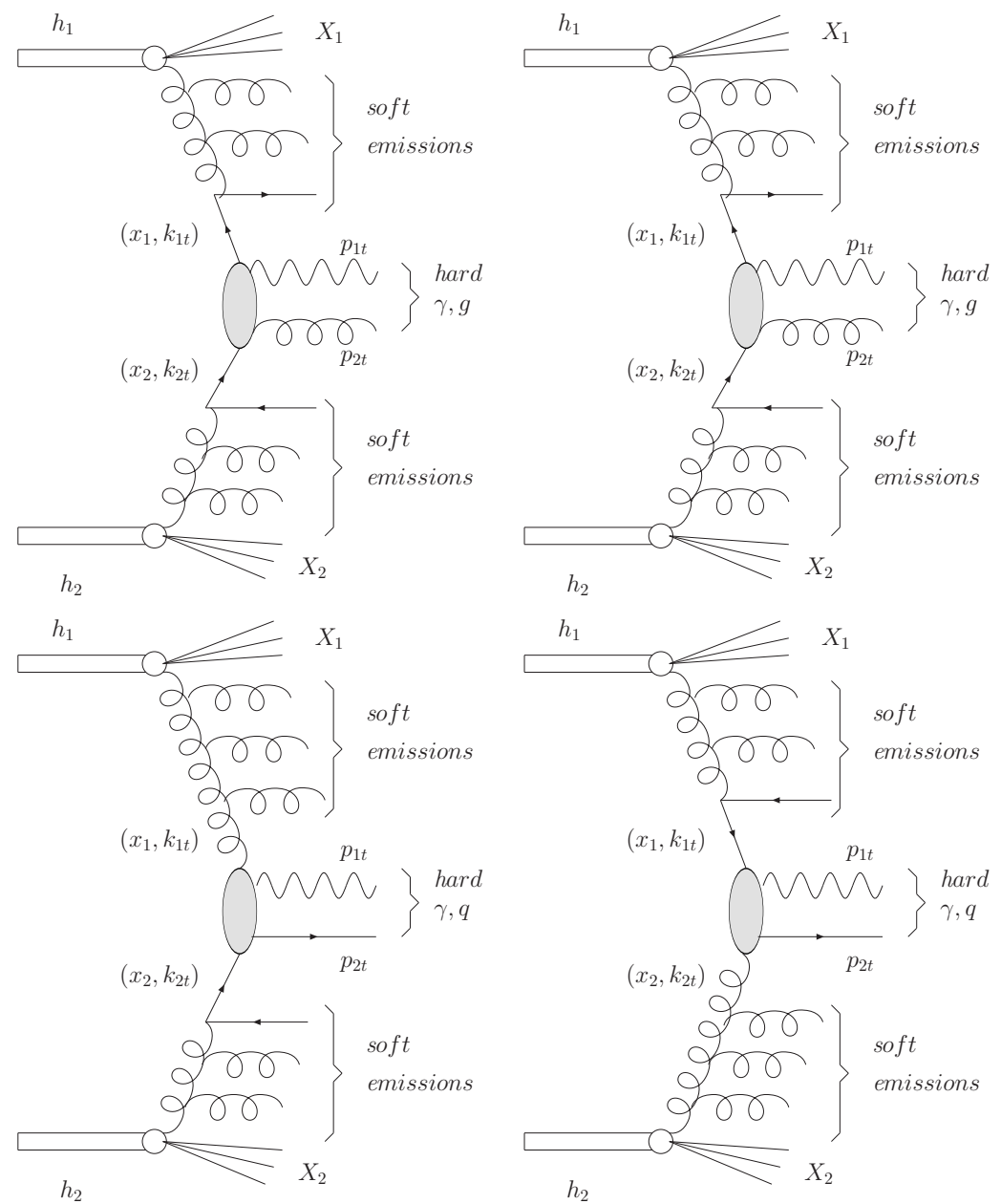

Figure 3: Diagrams for $k_{t}$-factorization approach to photon-jet correlations.

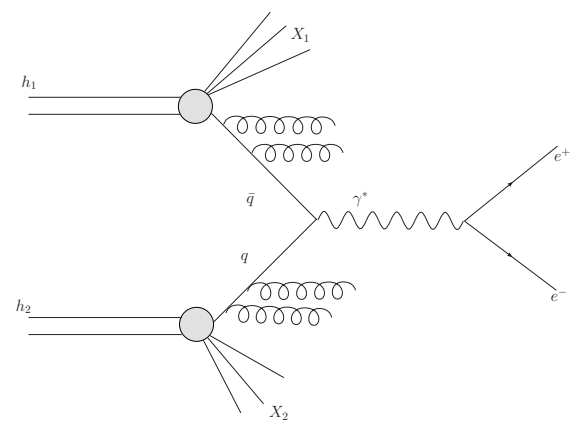

Figure 4: Leading-order diagram for $k_{t}$-factorization approach to Drell-Yan dilepton production. 


$$
x_{2}=\frac{m_{1, t}}{\sqrt{s}} \mathrm{e}^{-y_{1}}+\frac{m_{2, t}}{\sqrt{s}} \mathrm{e}^{-y_{2}},
$$

and $m_{1, t}$ and $m_{2, t}$ are so-called transverse masses defined as $m_{i, t}=\sqrt{p_{i, t}^{2}+m^{2}}$, where $m$ is the mass of the elementary particle. In the following we shall assume that all partons are massless. The objects denoted by $\mathscr{F}_{i}\left(x_{1}, k_{1, t}^{2}\right)$ and $\mathscr{F}_{j}\left(x_{2}, k_{2, t}^{2}\right)$ in the equation above are the unintegrated parton distributions in hadron $h_{1}$ and $h_{2}$, respectively. They are functions of longitudinal momentum fraction and transverse momentum of the incoming (virtual) parton. The sum is over all parton combinations leading to final particles $k$ and $l$.

In Fig.1 I show the leading diagrams for $c \bar{c}$ production. The heavy quark production is considered as a flag process for $k_{t}$-factorization approach. In Fig.2 I show the diagrams included for dijet correlations in Ref. [2]. In Fig.3 I show similar diagrams included for photon-jet correlations in Ref. [3]. Finally in Fig.4 I show basic leading-order diagram for Drell-Yan processes [4]. In the case of dilepton-jet correlations one needs to include also higher-order processes (see [4]).

The formula (2.1) allows to study different types of correlations. Here I shall limit to only few examples for dijet and photon-jet correlations. More examples for $c \bar{c}$ were discussed by $\mathbf{M}$. Łuszczak [5] and by G. Ślipek for Drell-Yan processes [6] at this workshop. The details concerning unintegrated gluon (parton) distributions can be found in original publications (see e.g.[1] and references therein).

\section{Selected results}

Let us start with dijet correlations. As an example in Fig.5 we show two-dimensional maps of the cross section in $\left(p_{1, t}, p_{2, t}\right)$ for processes shown in Fig.2. Only very few approaches in the literature include both gluons and quarks and antiquarks. In the calculation above we have used Kwieciński UPDFs with exponential nonperturbative form factor ${ }^{1}\left(b_{0}=1 \mathrm{GeV}^{-1}\right)$, and the factorization scale $\mu^{2}=\left(p_{t, \min }+p_{t, \max }\right)^{2} / 4=100 \mathrm{GeV}^{2}$.

The second example is for photon-jet correlations. In Fig.6 we show the effect of the scale evolution of the Kwieciński UPDFs on the azimuthal angle correlations between the photon and the associated jet. We show results for different initial conditions $\left(b_{0}=0.5,1.0,2.0 \mathrm{GeV}^{-1}\right)$. At the initial scale (fixed here as in the original GRV [3] to be $\mu^{2}=0.25 \mathrm{GeV}^{2}$ ) there is a sizable difference of the results for different $b_{0}$. The difference becomes less and less pronounced when the scale increases. At $\mu^{2}=100 \mathrm{GeV}^{2}$ the differences practically disappear. This is due to the fact that the QCD-evolution broadening of the initial parton transverse momentum distribution is much bigger than the typical initial nonperturbative transverse momentum scale.

Until now at the RHIC only photon-hadron correlation were measured by the PHENIX collaboration [7]. I show corresponding preliminary results of our calculation in Fig.7. The results are shown for different intervals of $p_{T, t i g}$ (photon) and $p_{T, \text { assoc }}$ (charged hadron). The intervals correspond to the experimental cuts. This calculation requires inclusion of fragmentation functions (see Fig.8). We have included both charged pions and charged kaons in this calcultion. It was as-

${ }^{1} F(b)=\exp \left(-b^{2} / 4 b_{0}^{2}\right)$ multiplies UPDFs in the impact parameter space and is responsible for nonperturbative effects included in addition to perturbative effects embedded in the Kwieciński evolution equations (for more details see e.g. [1]). 

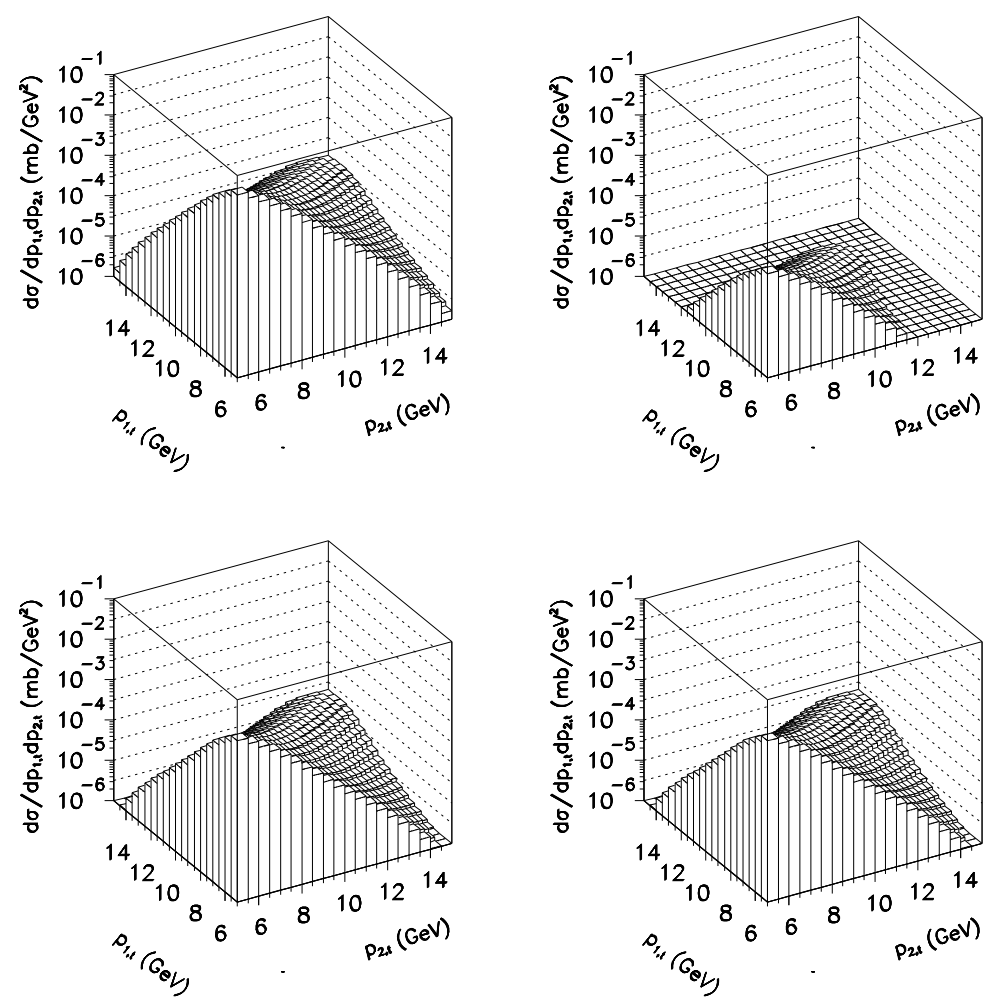

Figure 5: Two-dimensional distributions in $p_{1, t}$ and $p_{2, t}$ for different subprocesses $g g \rightarrow g g$ (upper left) $g g \rightarrow q \bar{q}$ (upper right), $g q \rightarrow g q$ (lower left) and $q g \rightarrow q g$ (lower right). In this calculation $\sqrt{s}=200 \mathrm{GeV}$ and Kwieciński UPDFs with exponential nonperturbative form factor $\left(b_{0}=1 \mathrm{GeV}^{-1}\right)$ and $\mu^{2}=100 \mathrm{GeV}^{2}$ were used. Here integration over full range of parton rapidities was made.

sumed that pions/kaons are emitted parallel to the jet (parton) direction. The details will be shown elsewhere. The distributions for different windows of transverse momenta are rather similar.

\section{Conclusions}

Motivated by the recent experimental results of hadron-hadron correlations at RHIC I have discussed $c \bar{c}$, jet-jet, photon-jet and $e^{+} e^{-}$correlations.

In comparison to recent works on dijet production in the framework of $k_{t}$-factorization approach, we have included two new mechanisms based on $g q \rightarrow g q$ and $q g \rightarrow q g$ hard subprocesses. This was done using the Kwieciński unintegrated parton distributions. We find that the new terms give significant contribution at RHIC energies. In general, the results of the $k_{t}$-factorization approach depend on UGDFs/UPDFs used, i.e. on approximation and assumptions made in their derivation.

In the region of small transverse momenta of outgoing particles the $k_{t}$-factorization approach is a good and efficient tool for the description of correlations [2]. Rather different results are obtained 


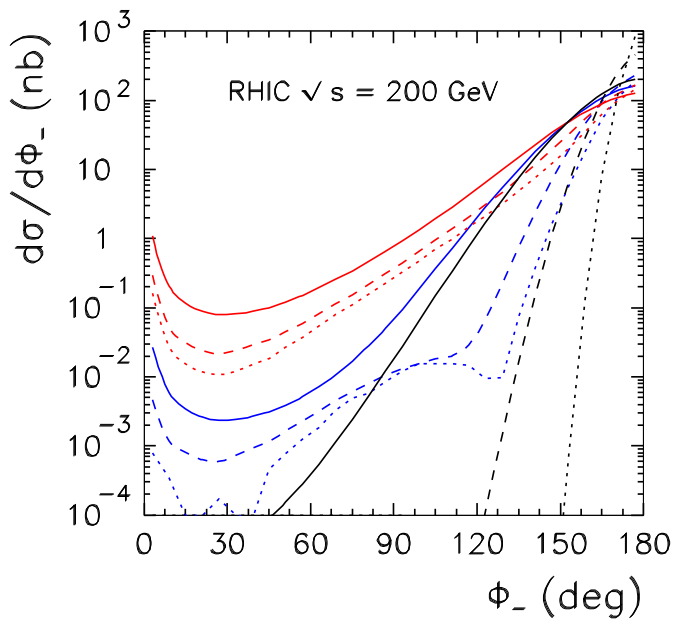

Figure 6: (Color online) Azimuthal angle correlation functions at RHIC energies for different scales and different values of $b_{0}$ of the Kwieciński distributions. The solid line is for $b_{0}=0.5 \mathrm{GeV}^{-1}$, the dashed line is for $b_{0}=1 \mathrm{GeV}^{-1}$ and the dotted line is for $b_{0}=2 \mathrm{GeV}^{-1}$. Three different values of the scale parameters are shown: $\mu^{2}=0.25,10,100 \mathrm{GeV}^{2}$ (the bigger the scale the bigger the decorellation effect, different colors on line). In this calculation $p_{1, t}, p_{2, t} \in(5,20) \mathrm{GeV}$ and $y_{1}, y_{2} \in(-5,5)$.

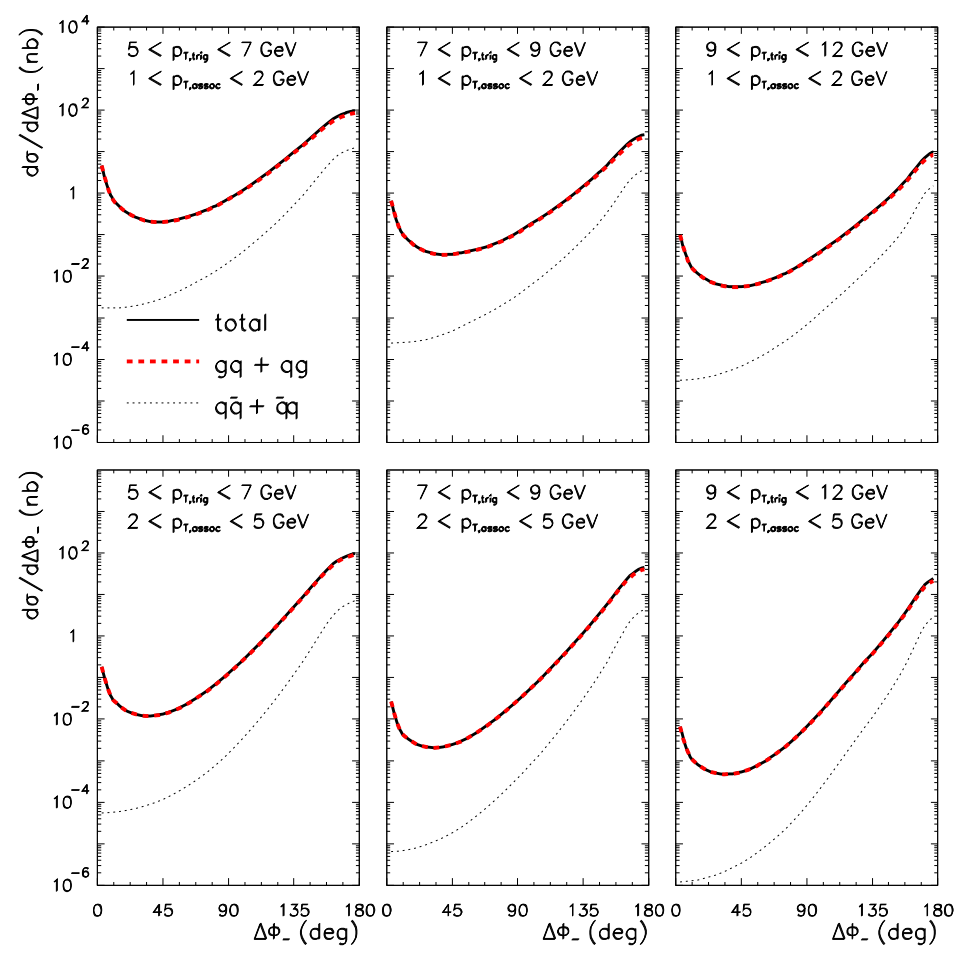

Figure 7: Photon-hadron azimuthal correlations for different windows of transverse momentum of the photon $\left(p_{T, \text { trig }}\right)$ and charged pion and kaon $\left(p_{T, \text { assoc }}\right)$. In this calculation $W=200 \mathrm{GeV}$. 


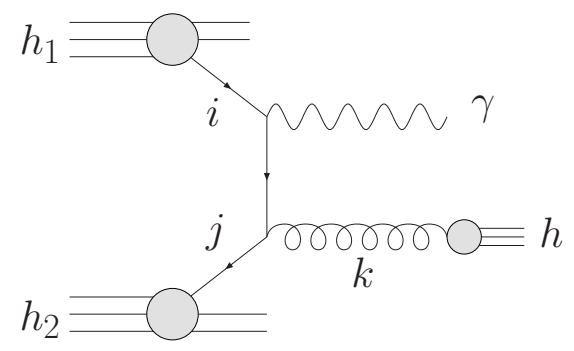

Figure 8: A schematic diagram illustrating associated production of photon and hadron.

with different UGDFs which opens a possibility to verify them experimentally. Consequences for particle-particle correlations, measured recently at RHIC, require a separate dedicated analysis.

We have discussed also photon-jet correlation observables. Up to now such correlations have not been studied experimentally. As for the dijet case we have concentrated on the region of small transverse momenta (semi-hard region) where the $k_{t}$-factorization approach seems to be the most efficient and theoretically justified tool. We have calculated correlation observables for different unintegrated parton distributions from the literature. Our previous analysis of inclusive spectra of direct photons suggests that the Kwieciński distributions give the best description at low and intermediate energies. We have discussed the role of the evolution scale of the Kwieciński UPDFs on the azimuthal correlations. In general, the bigger the scale the bigger decorrelation in azimuth is observed. When the scale $\mu^{2} \sim p_{t}^{2}$ (photon) $\sim p_{t}^{2}$ (associated jet) (for the kinematics chosen $\mu^{2} \sim 100 \mathrm{GeV}^{2}$ ) is assumed, much bigger decorrelations can be observed than from the standard Gaussian smearing prescription often used in phenomenological studies.

At RHIC one can measure jet-hadron correlations only for not too high transverse momenta of the trigger photon and of the associated hadron. This is precisely the semihard region discussed here. In this case the theoretical calculations require inclusion of the fragmentation process. This was done assuming independent parton fragmentation method with the help of fragmentation functions taken from the literature.

All the correlation observables have been studied for RHIC or even lower energies. This can be repeated in the future for LHC energies.

\section{References}

[1] M. Łuszczak and A. Szczurek, Phys. Rev. D73 (2006) 054028.

[2] A. Szczurek, A. Rybarska and G. Ślipek, Phys. Rev. D76 (2007) 034001.

[3] T. Pietrycki and A. Szczurek, Phys. Rev. D76 (2007) 034003.

[4] G. Ślipek and A. Szczurek, arXiv:0808.1360.

[5] M. Łuszczak, these proceedings.

[6] G. Ślipek, these proceedings.

[7] D.J. Kim, (PHENIX collaboration), these proceedings. 